\title{
Repeatability, variability and reference values of pulsed wave Doppler echocardiographic measurements in healthy Saanen goats
}

\author{
Aurélia A Leroux ${ }^{1 *}$, Frédéric Farnir ${ }^{2}$, Marie L Moonen ${ }^{3}$, Charlotte F Sandersen ${ }^{1}$, Stefan Deleuze ${ }^{1}$ and Hélène Amory ${ }^{1}$
}

\begin{abstract}
Background: Pulsed wave (PW) Doppler echocardiography has become a routine non invasive cardiac diagnostic tool in most species. However, evaluation of intracardiac blood flow requires reference values, which are poorly documented in goats. The aim of this study was to test the repeatability, the variability, and to establish the reference values of PW measurements in healthy adult Saanen goats. Using a standardised PW Doppler echocardiographic protocol, 10 healthy adult unsedated female Saanen goats were investigated three times at one day intervals by the same observer. Mitral, tricuspid, aortic and pulmonary flows were measured from a right parasternal view, and mitral and aortic flows were also measured from a left parasternal view. The difference between left and right side measurements and the intra-observer inter-day repeatability were tested and then the reference values of PW Doppler echocardiographic parameters in healthy adult female Saanen goats were established.

Results: As documented in other species, all caprine PW Doppler parameters demonstrated a poor inter-day repeatability and a moderate variability. Tricuspid and pulmonary flows were best evaluated on the right side whereas mitral and aortic flows were best obtained on the left side, and reference values are reported for healthy adult Saanen goats.

Conclusions: PW Doppler echocardiography allows the measurement of intracardiac blood flow indices in goats. The reference values establishment will help interpreting these indices of cardiac function in clinical cardiac cases and developing animal models for human cardiology research.
\end{abstract}

Keywords: Goat, Pulsed wave, Doppler echocardiography, Reference intervals, Repeatability, Variability

\section{Background}

During the last thirty years, pulsed wave (PW) Doppler echocardiography has been developed first in humans $[1,2]$, and then in several domestic animals species including dogs [3,4], cats [5], horses [6,7], cattle [8] and sheep [9]. This technique has become a routine for the diagnosis and evaluation of heart disease in veterinary medicine [10]. It allows detecting the returning signal during a time interval specified by a sample depth ignoring all other signals. Blood cells flow moving around the chosen specific location is analysed and gives information about direction,

\footnotetext{
* Correspondence: aurelia.leroux@ulg.ac.be

${ }^{1}$ Equine Clinic, Department of Companion Animals and Equids, Faculty of Veterinary Medicine, University of Liege, Boulevard de Colonster 20, Bât B41, Liege, Sart Tilman, Belgium

Full list of author information is available at the end of the article
}

velocity, character and timing of the blood flow which cannot be assess without Doppler imaging $[2,10]$. So it provides a non-invasive tool to evaluate intracardiac blood flow, to diagnose regurgitant flow through the cardiac valves and intracardiac shunts and to assess systolic and diastolic function of the heart $[10,11]$. Accurate interpretation of Doppler echocardiographic variables requires reference values following standardised measurement guidelines in the studied species to interpret indices of cardiac function [10].

Goats are animals easy to handle with a body and heart size comparable to that of humans. This makes the goat an attractive candidate for the development of animal models for human cardiology research, especially chronic models relying on measurements in awake or exercising animals [12,13]. Time-motion mode (M-mode), two-

\section{Biomed Central}


dimensional (2D) and colour-flow Doppler echocardiography has been demonstrated to be feasible in Philippine native goats [14] and in a pygmy goat [15]. More recently, M-mode and 2D-echocardiographic reference values have been established in awake healthy Saanen goats after repeatability and variability studies [16] and the effect of general anaesthesia in Saanen goats was studied using 2Dand colour Doppler echocardiography [17]. By contrast, no reference values of PW Doppler parameters were reported in goats. Only one study devoted to evaluate the cardiovascular changes induced by pregnancy and lactation reported PW Doppler values in Swedish adult domestic goats [18]. However, only aortic flow measurements were obtained and repeatability of these measurements was not determined in this study.

The aim of the present study was to test the variability, to establish the best technique to evaluate intracardiac blood flow and to establish the reference values of blood flow PW Doppler measurements in unsedated standing adult Saanen goats.

\section{Methods}

This experimental protocol was a part of a larger study and followed the guidelines of ethics of University of Liege. The reference number from the Ethical Committee was 655 accepted on the $1^{\text {st }}$ of June 2007.

\section{Animals}

Ten adult nulliparous female Saanen goats, aged 22 to 28 months (mean age: $24.7 \pm 2.1$ months) and weighing 51 to $80 \mathrm{~kg}$ (mean body weight: $65.1 \pm 8.3 \mathrm{~kg}$ ) were studied. All animals were considered to be healthy based on the history and the absence of abnormalities on physical examination, cardiac auscultation, electrocardiography (ECG), haematology, measurement of the serum haptoglobin, fibrinogen, total protein content, and the electrophoresis of serum proteins. A complete colour and PW Doppler echocardiography (from right and left side) was also performed in each goat before starting the protocol to be sure that the studied goats were free of any cardiac disease, including any valvulopathy. All the animals were fully accustomed to be handled. Before imaging, the hair was shaved on both sides, from the $3^{\text {rd }}$ to the $5^{\text {th }}$ right intercostal space just caudal to the triceps muscle mass, from 3 to $5 \mathrm{~cm}$ below the right olecranon to 5 to $10 \mathrm{~cm}$ above it. The shaved areas were then copiously rinsed with water and acoustic coupling was obtained using ultrasound gel.

\section{Echocardiographic protocol}

In the 10 studied goats, the PW Doppler echocardiographic protocol was repeated 3 times at one-day intervals. An ultrasound system (Vivid i, Software version 9.1.0, General Electric Healthcare Europe GMBH, Diegem,
Belgium) equipped with colour flow mapping and spectral Doppler mode and with a 1.5-3.6 MHz phased array transducer (GE 3S-RS probe, General Electric Healthcare Europe GMBH, Diegem, Belgium) was used to perform the PW Doppler echocardiography. All examinations and measurements were performed by the same observer (AAL), who had 3 full years expertise in large animal echocardiography (equids, cattle and goats), and were recorded digitally. Recordings were identified using number codes chosen by an external observer. All measurements were blindly performed off line by the same observer (AAL) using specific software (Echo Pac System for Vivid i, Software version 108.1.5, General Electric Healthcare Europe GMBH, Diegem, Belgium). All variables were measured 5 times on 5 different non consecutive cycles and an average of these 5 measurements was calculated for each variable. The cardiac cycle was chosen when the image quality of the flow was optimal. Examinations were performed on standing animals with the right or the left forelimb extended by an assistant as far forward as tolerated by the goat. Heart rate (HR) was calculated from the ECG tracings from 5 successive cardiac cycles during the echocardiographic recordings. Echocardiographic measurements were only performed when HR was less than 120 beats per minute to obviate a stress effect. A colour-flow Doppler echocardiography was performed each day on each measured intracardiac flow to confirm absence of any abnormal shunt.

Terminology and 2D-image orientation recommended by the Echocardiography Committee of The Specialty of Cardiology, American College of Veterinary Internal Medicine [19], were used. Goats were first examined from the left hemi thorax to assess the aortic and mitral blood flow. The transducer was placed in the $4^{\text {th }}$ intercostal space and adjusted to obtain a long axis four chamber view in 2D mode. From this view, the transducer was angled to optimize spatial alignment between the transducer and the mitral blood flow and the PW mode was selected. The sample volume $(4.91 \mathrm{~mm})$ was placed between the mitral valve leaflets opening in diastole and the mitral inflow velocity spectrum was recorded (Figure 1). From the initial 2D left parasternal long axis four chambers view, the transducer was then slightly turned forwards to obtain a standard long axis five chamber view. The aortic flow velocity spectrum was obtained by placing the sample volume of the PW mode just downstream from the aortic valve in the aortic root (Figure 2).

The goats were then examined from the right hemi thorax. A right parasternal long axis four chambers view with chordae tendinae and mitral valve clearly visible was obtained in 2D-mode. This view was then tilted as ventrally as possible to obtain the most optimal possible alignment between the transducer and the mitral flow. In 


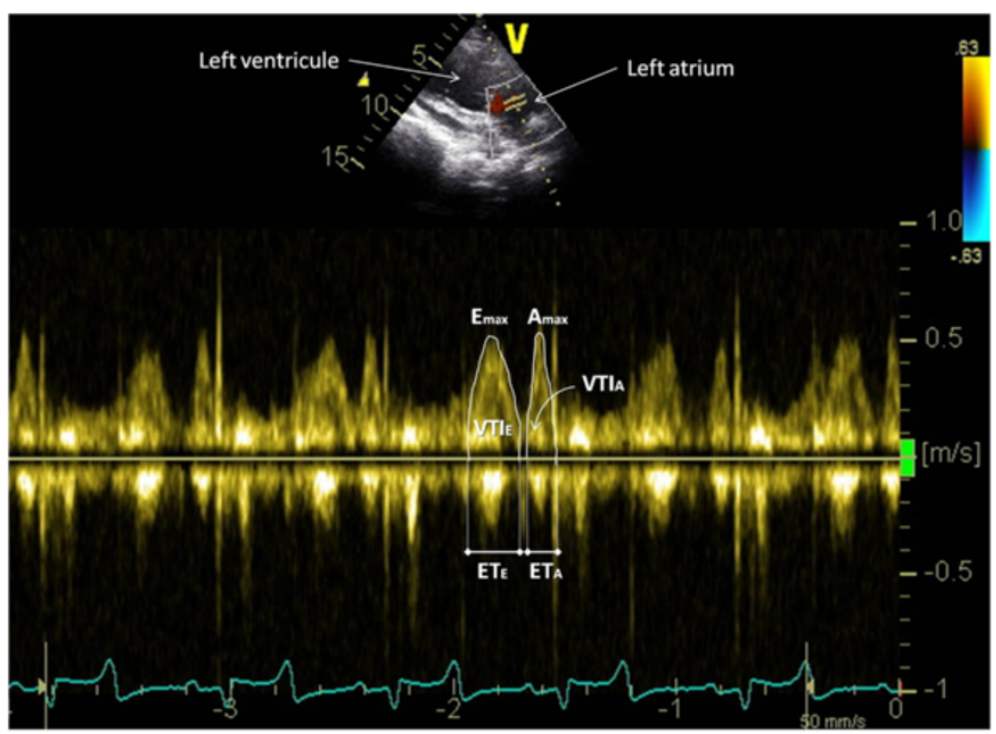

Figure $1 \mathrm{Mitral}$ inflow velocity spectrum obtained from a left parasternal long axis four chamber view. The peak early filling velocity $\left(E_{\text {max }}\right)$, the peak filling velocity of the transmitral flow during atrial contraction $\left(A_{\max }\right)$, the E-peak and A-peak velocity time integral $\left(V T I_{E}\right.$ and $V T I_{A}$ respectively) and the E-peak and $A$-peak ejection times ( $E T_{E}$ and $E T_{A}$ respectively) were measured as shown.

PW-mode, the sample volume was placed in the left ventricle just between the mitral valve leaflets opening in diastole to record the mitral inflow velocity spectrum. Then, the transducer was angled to optimize spatial alignment between the transducer and the tricuspid flow and the sample volume was placed in the right ventricle just between the tricuspid valve leaflets opening in diastole to record the tricuspid inflow velocity spectrum (Figure 3 ). Starting from the right parasternal 2D four chambers view, the transducer was then rotated slightly cranially to obtain a right parasternal long axis view of the left ventricular outflow tract and to measure the aortic internal diameter at the basis of the valves (Ao) at end diastole. From this five chambers view, the beam was tilted in order to obtain an optimal alignment with aortic blood flow. The PW mode was selected and aortic flow velocity spectrum was obtained by placing the sample volume in the aortic root just distal to the valves. The pulmonary artery diameter at

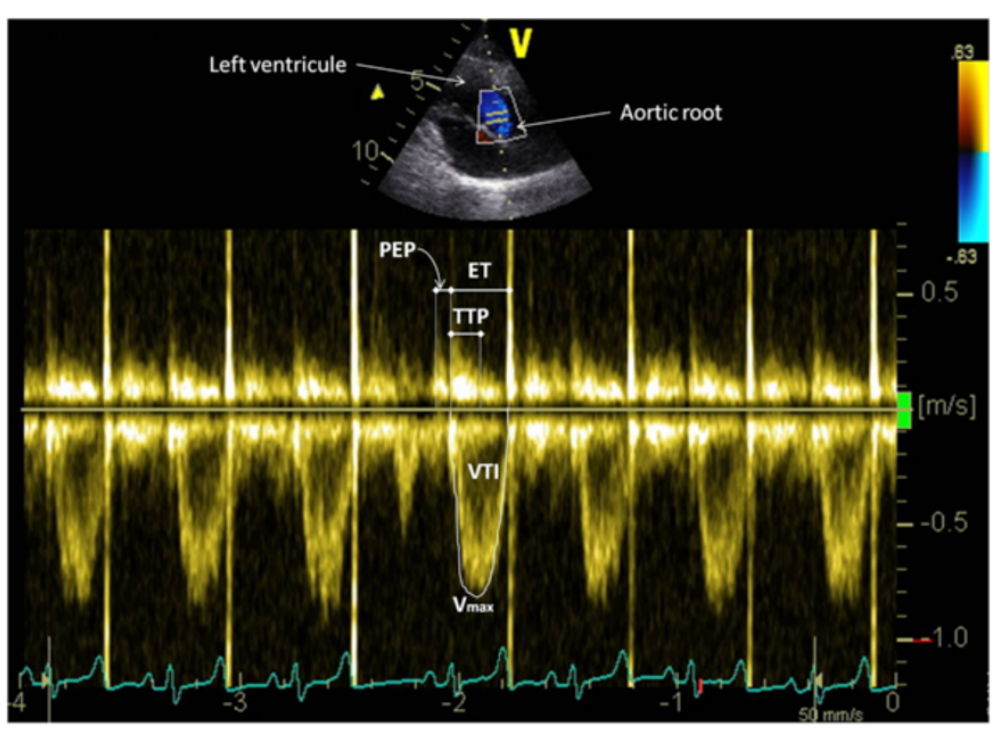

Figure 2 Aortic flow velocity spectrum obtained from a left parasternal long axis five chamber view. The peak velocity of the aortic flow $\left(V_{\text {max }}\right)$, the velocity time integral $(\mathrm{VTI})$, the ejection time (ET), the time to peak velocity (TTP) and the pre-ejection period (PEP) of the aortic flow were measured as shown. 


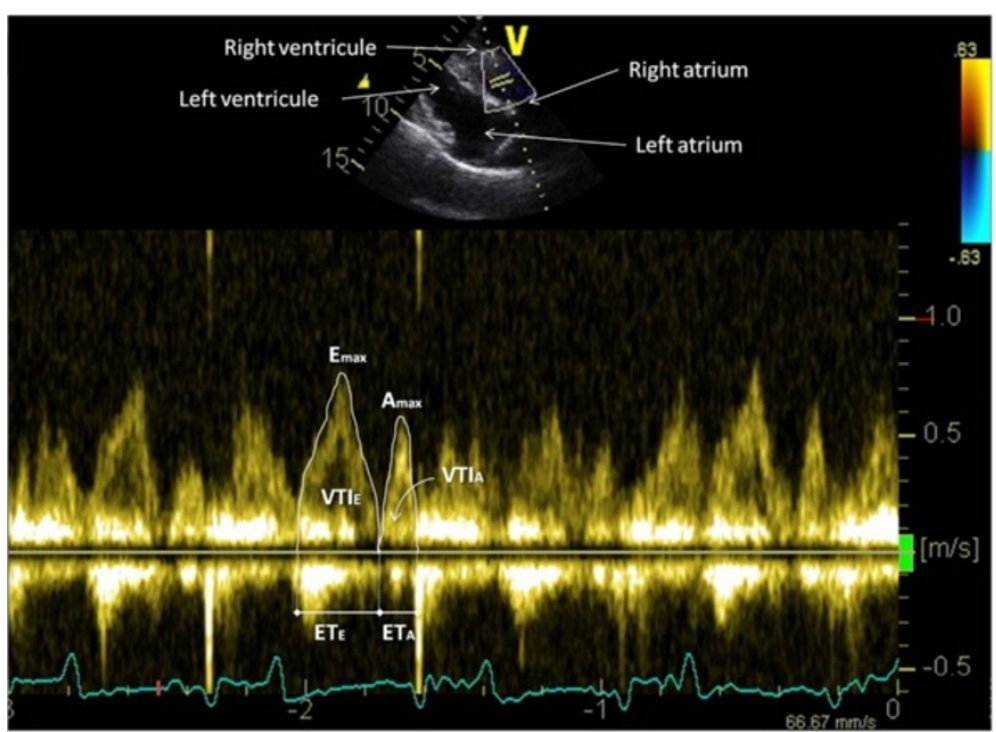

Figure 3 Tricuspid inflow velocity spectrum obtained from a tilted right parasternal long axis four chamber view. The peak early filling velocity $\left(E_{\max }\right)$, the peak filling velocity of the tricuspid flow during atrial contraction $\left(A_{\text {max }}\right)$, the E-peak and A-peak velocity time integral $\left(V T I_{E}\right.$ and $V T I_{A}$ respectively) and the E-peak and A-peak ejection times (ET $T_{E}$ and $E T_{A}$ respectively) were measured as shown.

end diastole was measured $(\mathrm{Pu})$ from a right parasternal right ventricular inflow-outflow view obtained by directing the transducer slightly cranio-dorsally and by placing it one intercostal space forwards. PW-mode was selected and the sample volume was positioned just distal to the pulmonary valves to assess the pulmonary outflow velocity spectrum (Figure 4).

\section{Doppler measurements and calculations}

From the mitral and tricuspid velocity spectral recordings, the peak velocity during the early ventricular filling called $\mathrm{E}$ peak and during the atrial contraction called $\mathrm{A}$ peak were measured ( $E_{\max }$ and $A_{\max }$ respectively). The areas under the two velocity peaks (VTI of E peak and of A peak) were measured by manually tracing the

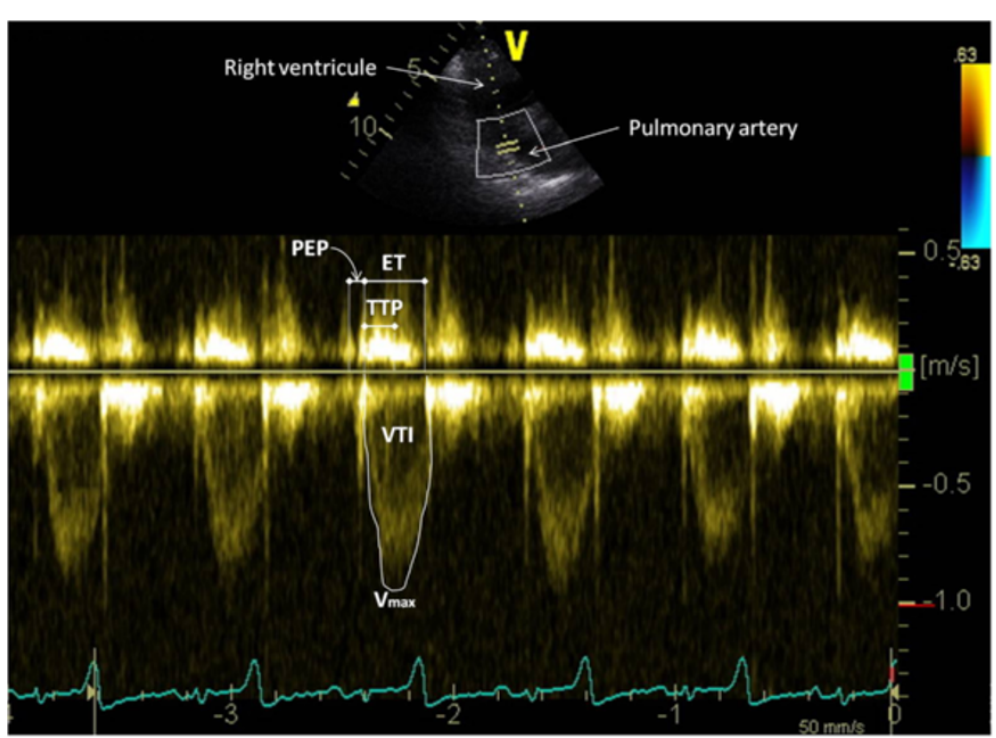

Figure 4 Pulmonary outflow velocity spectrum obtained from a right parasternal right ventricular inflow-outflow view. The peak velocity of the pulmonary flow $\left(\mathrm{V}_{\max }\right)$, the velocity time integral $(\mathrm{VTI})$, the ejection time (ET), the time to peak velocity (TTP) and the pre-ejection period (PEP) of the pulmonary flow were measured as shown. 
modal velocity envelope of the Doppler signal. This allowed the assessment of the mean velocity of the blood flow during the early ventricular filling $\left(\mathrm{E}_{\text {mean }}\right)$ and during the atrial contraction $\left(\mathrm{A}_{\text {mean }}\right)$. The ejection time (ET) of the E peak and of the A peak were measured from the onset to the end of each peak. The deceleration (Dec) time and slope of the early filling velocity were measured by tracing the slope between the maximal $\mathrm{E}$ peak velocity and the return to baseline at the end of the flow profile. From $E_{\max }$ and $A_{\max }$, the ratio $E_{\max } / A_{\max }$ was calculated.

From the aortic and pulmonary velocity spectral recordings, the peak velocity of the blood flow $\left(\mathrm{V}_{\max }\right)$ was measured by placing the cursor at the maximal point of the blood flow profile. The area under the velocity waveform (VTI) was measured by manual tracing of the modal velocity envelope of the flow profile, thus allowing the measurement of the mean velocity of blood flow $\left(\mathrm{V}_{\text {mean }}\right)$. The ejection time (ET) was measured from the onset to the end of the spectral waveform, the time to peak (TTP) was measured from the onset of the Doppler waveform to the beginning of the maximum velocity plateau, the pre-ejection period (PEP) was measured from the onset of the QRS complex to the onset of the spectral waveform, and the Dec time was calculated by subtracting TTP from ET. The acceleration (Acc) and Dec slope of the flow profile were measured by tracing the slope from the onset to the peak and from the peak to the end of the velocity spectral waveform, respectively. From the measured parameters, the ratio PEP/ET was calculated and the stroke volume (SV) and the cardiac output $(\mathrm{CO})$ were obtained using the following standard formulae:

$$
\begin{aligned}
& S V=\pi . V T I .(A o / 2)^{2} \\
& C O=S V . H R .
\end{aligned}
$$

The stoke index (SI) and the cardiac index (CI) were calculated by dividing $\mathrm{SV}$ and $\mathrm{CO}$, respectively, by the body weight.

\section{Repeatability and statistical analysis}

In all goats, the echocardiographic protocol was repeated 3 times at one-day intervals by the same observer (AAL). Within each day, each variable was measured 5 times on 5 different non consecutive cardiac cycles. Observed means, standard deviations (SD), least square means, and standard errors (SE) were calculated for each variable. For measurements of tricuspid and pulmonary flows, a two-way ANOVA considering goats, days and interaction between goats and days as factors, and, for measurements of mitral and aortic flows, a three-way ANOVA considering goats, days, side of echocardiography and interaction between these different variables as factors, were performed and allowed the determination of the intra-observer inter-day repeatability of the measurements. A $P$-value less than 0.05 was considered significant. The within-goat within-day variability was evaluated using the coefficient of variation (Within-day $\mathrm{CV}$ ) measured from standard errors and observed means obtained from the two-way ANOVA for each variable. The within-goat between-day variability (Between-day $\mathrm{CV}$ ), i.e. the variability of the same repeated measurements on the same goat independently of the day, was evaluated from standard deviations and observed means obtained in a one way ANOVA considering only goats as factor. Degree of variability of each measurement was defined arbitrarly as follows: variables with a CV inferior to $10 \%$ were considered to have low variability, those with a CV between $10 \%$ and $20 \%$ were considered to have moderate variability, and those with a CV superior to $20 \%$ were considered to have high variability. In addition to the $\mathrm{CV}$, absolute variability was obtained by calculating confidence interval for each variable. This interval corresponds to the interval within which the real value of the measurement has a $95 \%$ chance to be included. Superior and inferior limits of this interval were obtained as follows: observed mean $+1.96 \mathrm{xSE}$ and observed mean - 1.96xSE.

For each variable, the mean, the SD, and the range of values for all these measurements were used to establish the reference values of PW Doppler parameters in unsedated adult healthy female Saanen goats.

\section{Results}

The mean HR during the echocardiographic examination was $92.75 \pm 11.18$ beats/minute and ranged from 75 to 120 beats/minute. The image quality and the Doppler spectra were good in all goats, except for the quality of the tricuspid flow and the right side aortic flow which were often poor. Moreover, obtaining a good quality $2 \mathrm{D}$ right parasternal view of the heart base at the level of the pulmonary valves before to shift in PW-mode appeared to be sometimes difficult. For this view, the transducer had to be advanced far forward under the forelimb, which required an assistant pulling the right forelimb forward and upward during the examination.

The least square mean value and the standard error to the mean of each blood flow measurements obtained on day 1, day 2 and day 3 and the multivariable ANOVA test were calculated to evaluate the repeatability of these measurements. Concerning the aortic flow, significant between days differences were observed for $\mathrm{V}_{\text {max }}$, VTI, AccTime, CO and CI. Almost all measurements of the pulmonary flow were significantly different between days except PEP and PEP/ET. Mitral and tricuspid flow velocities $\left(E_{\text {max }}, E_{\text {mean }}, A_{\text {max }}, A_{\text {mean }}, E_{\text {max }} / A_{\text {max }}\right)$ and $E$ 
peak and A peak mitral and tricuspid VTI were significantly different between days.

Comparisons of the PW Doppler echocardiographic measurements of the aortic and mitral flows obtained from the right and from the left side are shown in Tables 1 and 2 respectively. For the aortic flow, only PEP and PEP/ET were not significantly different whilst all other parameters were significantly different from the right and the left side. Most of the parameters, especially $\mathrm{V}_{\max }$ and $\mathrm{V}_{\text {mean }}$, were significantly higher when they were obtained from the left side than from the right side. For mitral flow, all parameters of A peak but only ET of $E$ peak were not significantly different from both sides. The mitral $E_{\max }, E_{\text {mean }}$ and VTI of E peak were higher when obtained from the left side than when obtained from the right side.

The variability of all the PW Doppler measurements was evaluated on the basis of the within-day and between-day $\mathrm{CV}$ and of the calculation of confidence intervals. The results of the measurements of the aortic, mitral, pulmonary and tricuspid flows are shown in Tables 1, 2, 3 and 4, respectively. Most of the parameters had low to moderate variability excepted for TTP of the aortic flow, acc slope of the aortic and pulmonary flows, and Dec Time and Dec slope of the mitral and tricuspid E peaks that showed a high variability. For all flows, within-day variability was clearly lower than betweenday variability.

\section{Discussion}

The results of this paper show that good quality PW Doppler flow spectrum of each cardiac valve can be obtained on standing unsedated goats like in most other animal species. The effects of the physiological factors such as body size [20], breed [21], age [22], lactation [18] and pregnancy $[18,23]$, which are known to affect PW Doppler measurements were not investigated in the present study. A colour-flow Doppler echocardiography performed each day before each PW-Doppler echocardioghraphic protocol did not reveal any abnormality. Particularly, any suspicion of interatrial communication or patent foramen ovale such as reported in 9 healthy Saanen goats [17] could be excluded.

To assess a pulmonary spectrum flow in this study, the transducer had to be advanced far cranially under the forelimb, which required an assistant pulling the right forelimb forward and upward during the examination. This procedure was not well tolerated by all of the investigated animals, and implies than the investigated goat is fully accustomed to being handled, which is not always the case in caprine field practice. Moreover, at this level, the cardiac window often appeared to be narrow as previously reported in goats [16,18]. But when a heart base view of sufficient quality was obtained, the recorded pulmonary outflow velocity spectrum was generally speaking of good quality, suggesting a good alignment of the transducer with the pulmonary blood flow,

Table 1 Comparison of pulse wave Doppler echocardiographic parameters of the aortic flow obtained on the right and on the left side in 10 healthy adult Saanen goats, and within-day and between-day variability of these measurements performed on 3 following days by the same observer

\begin{tabular}{|c|c|c|c|c|c|c|}
\hline & $\begin{array}{l}\text { LSMean } \pm \text { SE } \\
\text { Right side }\end{array}$ & $\begin{array}{l}\text { LSMean } \pm \text { SE } \\
\text { Left side }\end{array}$ & $\begin{array}{l}\text { Observed } \\
\text { Mean } \pm \text { SD }\end{array}$ & $\begin{array}{l}\text { Confidence Intervals } \\
(5-95 \%)\end{array}$ & $\begin{array}{l}\text { Within-day } \\
\text { CV (\%) }\end{array}$ & $\begin{array}{l}\text { Between-day } \\
\text { CV (\%) }\end{array}$ \\
\hline$V_{\max }(\mathrm{m} / \mathrm{s})$ & $0.99 \pm 0.006$ & $1.11 \pm 0.006^{*}$ & $1.05 \pm 0.14$ & $0.98-1.11$ & 4.87 & 6.62 \\
\hline$V_{\text {mean }}(\mathrm{m} / \mathrm{s})$ & $0.73 \pm 0.004$ & $0.79 \pm 0.004^{*}$ & $0.76 \pm 0.09$ & $0.72-0.81$ & 5.60 & 6.43 \\
\hline $\mathrm{ET}$ (ms) & $266.89 \pm 1.41$ & $271.95 \pm 1.40 *$ & $269.42 \pm 23.17$ & $254.38-284.45$ & 4.68 & 6.37 \\
\hline $\mathrm{VTI}(\mathrm{cm})$ & $19.58 \pm 0.14$ & $21.42 \pm 0.14 *$ & $20.51 \pm 2.77$ & $18.98-22.03$ & 6.76 & 8.47 \\
\hline PEP (ms) & $49.80 \pm 0.55$ & $50.89 \pm 0.55$ & $50.38 \pm 6.84$ & $44.48-56.28$ & 11.49 & 13.35 \\
\hline TTP (ms) & $102.53 \pm 1.57$ & $82.74 \pm 1.56$ * & $92.58 \pm 19.70$ & $75.77-109.37$ & 16.31 & 20.70 \\
\hline Acc slope $\left(\mathrm{m} / \mathrm{s}^{2}\right)$ & $10.28 \pm 0.24$ & $13.95 \pm 0.24$ * & $12.13 \pm 3.29$ & $9.58-14.67$ & 17.67 & 23.93 \\
\hline Dec slope $\left(\mathrm{m} / \mathrm{s}^{2}\right)$ & $6.35 \pm 0.08$ & $6.067 \pm 0.08 *$ & $6.21 \pm 1.44$ & $5.38-7.03$ & 11.14 & 15.16 \\
\hline Dec Time (ms) & $164.35 \pm 1.79$ & $189.21 \pm 1.78 *$ & $176.85 \pm 26.82$ & $157.68-196.01$ & 10.30 & 12.36 \\
\hline SV (ml) & $61.77 \pm 0.45$ & $67.61 \pm 0.45 *$ & $64.71 \pm 8.60$ & $59.87-69.55$ & 6.76 & 8.52 \\
\hline CO (I/min) & $5.80 \pm 0.44$ & $6.16 \pm 0.44 *$ & $5.98 \pm 0.96$ & $5.51-6.45$ & 6.76 & 8.46 \\
\hline $\mathrm{SI}(\mathrm{ml} / \mathrm{kg})$ & $0.96 \pm 0.007$ & $1.05 \pm 0.007 *$ & $1.00 \pm 0.14$ & $0.92-1.08$ & 6.76 & 8.98 \\
\hline $\mathrm{Cl}(\mathrm{ml} / \mathrm{kg} / \mathrm{min})$ & $89.81 \pm 0.72$ & $95.27 \pm 0.72 *$ & $92.45 \pm 14.91$ & $84.78-100.11$ & 6.76 & 8.82 \\
\hline PEP/ET & $0.19 \pm 0.003$ & $0.19 \pm 0.003$ & $0.19 \pm 0.03$ & $0.16-0.22$ & 13.37 & 9.46 \\
\hline
\end{tabular}

Acc: Acceleration, Cl: Cardiac Index, CO: Cardiac output, CV: Coefficient of variation, Dec: Deceleration, ET: Ejection time of the aortic flow, LSMean: Least Square Mean, PEP: Pre-ejection period of the aortic flow, SD: Standard deviation, SE: Standard error, SI: Stroke index, SV: Stroke volume, TPP: Time to peak of the aortic flow, $V_{\text {max }}$ : Peak velocity of the aortic flow, $V_{\text {mean: }}$ Mean velocity of the aortic flow, VTI: Velocity time integral of the aortic flow.

* Significantly different from corresponding value obtained from the right side, $p<0.05$. 
Table 2 Comparison of pulse wave Doppler echocardiographic parameters of the mitral flow obtained on the right and on the left side in $\mathbf{1 0}$ healthy adult Saanen goats, and within-day and between-day variability of these measurements performed on 3 following days by the same observer

\begin{tabular}{|c|c|c|c|c|c|c|}
\hline & $\begin{array}{l}\text { LSMean } \pm \text { SE } \\
\text { Right side }\end{array}$ & $\begin{array}{l}\text { LSMean } \pm \text { SE } \\
\text { Left side }\end{array}$ & $\begin{array}{l}\text { Observed } \\
\text { Mean } \pm \text { SD }\end{array}$ & $\begin{array}{l}\text { Confidence Intervals } \\
(5-95 \%)\end{array}$ & $\begin{array}{l}\text { Within-day } \\
\text { CV (\%) }\end{array}$ & $\begin{array}{l}\text { Between-day } \\
\text { CV (\%) }\end{array}$ \\
\hline \multicolumn{7}{|l|}{ E-Peak } \\
\hline $\mathrm{E}_{\max }(\mathrm{m} / \mathrm{s})$ & $0.57 \pm 0.005$ & $0.59 \pm 0.005^{*}$ & $0.58 \pm 0.04$ & $0.52-0.63$ & 7.23 & 10.66 \\
\hline$E_{\text {mean }}(\mathrm{m} / \mathrm{s})$ & $0.40 \pm 0.004$ & $0.42 \pm 0.004 *$ & $0.41 \pm 0.03$ & $0.37-0.45$ & 7.97 & 10.80 \\
\hline VTI (cm) & $8.84 \pm 0.10$ & $9.52 \pm 0.10 *$ & $9.18 \pm 0.97$ & $8.07-10.29$ & 10.52 & 13.81 \\
\hline ET (ms) & $224.57 \pm 2.52$ & $227.54 \pm 2.52$ & $226.06 \pm 22.17$ & $199.01-253.11$ & 9.91 & 13.65 \\
\hline Dec Time (ms) & $119.99 \pm 1.95$ & $104.99 \pm 1.95 *$ & $112.49 \pm 19.12$ & $91.56-133.41$ & 17.75 & 21.22 \\
\hline Dec slope $\left(\mathrm{m} / \mathrm{s}^{2}\right)$ & $5.17 \pm 0.20$ & $6.74 \pm 0.20 *$ & $5.96 \pm 1.45$ & $3.78-8.13$ & 22.35 & 41.53 \\
\hline \multicolumn{7}{|l|}{ A-Peak } \\
\hline$A_{\max }(m / s)$ & $0.51 \pm 0.005$ & $0.52 \pm 0.005$ & $0.51 \pm 0.05$ & $0.46-0.56$ & 10.24 & 11.50 \\
\hline$A_{\text {mean }}(\mathrm{m} / \mathrm{s})$ & $0.39 \pm 0.004$ & $0.39 \pm 0.004$ & $0.39 \pm 0.04$ & $0.34-0.43$ & 11.42 & 12.79 \\
\hline $\mathrm{VTI}(\mathrm{cm})$ & $4.27 \pm 0.06$ & $4.26 \pm 0.06$ & $4.27 \pm 0.64$ & $3.61-4.92$ & 14.98 & 17.43 \\
\hline ET (ms) & $110.74 \pm 1.44$ & $110.44 \pm 1.44$ & $110.59 \pm 14.76$ & $95.17-126.01$ & 13.26 & 15.90 \\
\hline $\mathrm{E}_{\max } / \mathrm{A}_{\max }$ & $1.12 \pm 0.01$ & $1.15 \pm 0.01$ & $1.14 \pm 0.11$ & $1.01-1.26$ & 9.31 & 12.35 \\
\hline
\end{tabular}

$A_{\text {max }}$ : peak velocity of the mitral flow during the atrial contraction, $A_{\text {mean }}$ : mean velocity of the mitral flow during the atrial contraction, $C V$ : Coefficient of variation, Dec: Deceleration, $E_{\max }$ : peak velocity of the mitral flow during the early ventricular filling, $E_{\text {mean }}$ : mean velocity of the mitral flow during the early ventricular filling, ET: Ejection time, LSMean: Least Square Mean, SD: Standard deviation, SE: Standard error, VTI: Velocity time integral.

* Significantly different from corresponding value obtained from the right side, $\mathrm{p}<0.05$.

as previously reported in horses [10,24]. On the contrary, the tilted 2D right parasternal long axis four chambers view was in most of the goats of very good quality, but the obtained flows at the level of the mitral and tricuspid valves were generally underwhelming, as described in horses [7]. Moreover, a true left parasternal apical view as used in dogs to measure aortic, mitral and sometimes tricuspid flows [10,25], was not possible to obtain in goats because of the presence of gas in the reticulo-rumen [18].

Table 3 Within-day and between-day variability and confidence interval of pulse wave Doppler echocardiographic measurements of the pulmonary flow in 10 healthy adult Saanen goats performed 3 times at 1 day interval by the same observer

\begin{tabular}{lcccc}
\hline & Observed Mean \pm SD & Confidence Intervals (5 - 95\%) & Within-day CV (\%) & Between-day CV (\%) \\
\hline$V_{\text {max }}(\mathrm{m} / \mathrm{s})$ & $0.99 \pm 0.12$ & $0.93-1.05$ & 5.33 & 6.19 \\
$V_{\text {mean }}(\mathrm{m} / \mathrm{s})$ & $0.76 \pm 0.10$ & $0.71-0.80$ & 5.49 & 6.58 \\
ET (ms) & $266.7 \pm 23.4$ & $254.45-279.04$ & 4.77 & 5.26 \\
VTI (cm) & $20.14 \pm 2.74$ & $19.02-21.25$ & 6.03 & 6.31 \\
PEP (ms) & $51.1 \pm 8.0$ & $44.89-57.39$ & 12.34 & 13.94 \\
TTP (ms) & $112.5 \pm 27.1$ & $95.57-129.44$ & 15.89 & 17.17 \\
Acc slope $\left(\mathrm{m} / \mathrm{s}^{2}\right)$ & $9.31 \pm 2.83$ & $7.35-11.27$ & 20.19 & 24.02 \\
Dec slope $\left(\mathrm{m} / \mathrm{s}^{2}\right)$ & $6.81 \pm 1.97$ & $5.71-7.91$ & 12.54 & 18.36 \\
Dec Time $(\mathrm{ms})$ & $154.3 \pm 31.9$ & $136.44-172.05$ & 12.48 & 13.17 \\
SV (ml) & $77.69 \pm 11.16$ & $73.41-81.97$ & 6.02 & 6.28 \\
CO (I/min) & $7.34 \pm 1.50$ & $6.93-7.74$ & 6.02 & 6.30 \\
SI (ml/kg) & $1.20 \pm 0.17$ & $1.13-1.27$ & 6.02 & 6.47 \\
Cl (ml/kg/min) & $113.31 \pm 22.88$ & $106.82-119.80$ & 6.02 & 6.53 \\
PEP/ET & $0.19 \pm 0.04$ & $0.16-0.22$ & 14.86 & 16.41
\end{tabular}

Acc: Acceleration, Cl: Cardiac index, CO: Cardiac output, CV: Coefficient of variation, Dec: Deceleration, ET: Ejection time of the pulmonary flow, PEP: Pre-ejection period of the pulmonary flow, SD: Standard deviation, SI: Stroke index, SV: Stroke volume, TPP: Time to peak of the pulmonary flow, $\mathrm{V}_{\text {max: }}$ Peak velocity of the pulmonary flow, $\mathrm{V}_{\text {mean: }}$ Mean velocity of the pulmonary flow, VTI: Velocity time integral of the pulmonary flow. 
Table 4 Within-day and between-day variability and confidence interval of pulse wave Doppler echocardiographic measurements of the tricuspid flow in $\mathbf{1 0}$ healthy adult Saanen goats performed $\mathbf{3}$ times at $\mathbf{1}$ day interval by the same observer

\begin{tabular}{|c|c|c|c|c|}
\hline & Observed Mean \pm SD & Confidence Intervals (5 - 95\%) & Within-day CV (\%) & Between-day CV (\%) \\
\hline \multicolumn{5}{|l|}{ E-Peak } \\
\hline$E_{\max }(\mathrm{m} / \mathrm{s})$ & $0.67 \pm 0.15$ & $0.60-0.73$ & 9.82 & 10.34 \\
\hline$E_{\text {mean }}(\mathrm{m} / \mathrm{s})$ & $0.49 \pm 0.12$ & $0.44-0.54$ & 10.11 & 10.88 \\
\hline $\mathrm{VTI}(\mathrm{cm})$ & $10.60 \pm 3.10$ & $9.14-12.06$ & 15.21 & 15.69 \\
\hline $\mathrm{ET}(\mathrm{ms})$ & $220.6 \pm 54.5$ & $196.34-244.77$ & 11.16 & 12.53 \\
\hline Dec Time (ms) & $108.9 \pm 34.7$ & $86.23-131.72$ & 22.03 & 23.81 \\
\hline Dec slope $\left(\mathrm{m} / \mathrm{s}^{2}\right)$ & $6.89 \pm 3.09$ & $5.02-8.74$ & 25.65 & 30.87 \\
\hline \multicolumn{5}{|l|}{ A-Peak } \\
\hline$A_{\max }(\mathrm{m} / \mathrm{s})$ & $0.58 \pm 0.12$ & $0.51-0.64$ & 10.70 & 12.29 \\
\hline$A_{\text {mean }}(\mathrm{m} / \mathrm{s})$ & $0.45 \pm 0.10$ & $0.39-0.49$ & 11.07 & 12.60 \\
\hline $\mathrm{VTI}(\mathrm{cm})$ & $5.11 \pm 1.24$ & $4.30-5.91$ & 16.20 & 17.97 \\
\hline $\mathrm{ET}(\mathrm{ms})$ & $117.3 \pm 24.2$ & $103.86-130.77$ & 11.41 & 13.08 \\
\hline$E / A$ & $1.16 \pm 0.19$ & $1.03-1.29$ & 11.14 & 12.35 \\
\hline
\end{tabular}

$A_{\text {max }}$ : peak velocity of the tricuspid flow during the atrial contraction, $A_{\text {mean }}$ : mean velocity of the tricuspid flow during the atrial contraction, $\mathrm{Cl}$ : Confidence Intervals, CV: Coefficient of variation, Dec: Deceleration, $\mathrm{E}_{\mathrm{max}}$ : peak velocity of the tricuspid flow during the early ventricular filling, $\mathrm{E}_{\mathrm{mean}}$ : mean velocity of the tricuspid flow during the early ventricular filling, ET: Ejection time, SD: Standard deviation, VTI: Velocity time integral.

The repeatability of the PW Doppler measurements in our study was poor, especially for Acc and Dec slopes of all intra-cardiac blood flows and, on the contrary to what could be expected on the basis of the good quality of the pulmonary flow spectrum, for all pulmonary flow measurements. PW Doppler measurements have been already described as poorly repeatable in other species $[10,26,27]$. Moreover, slopes of intra-cardiac blood flows are generally the less repeatable Doppler parameters [28]. Aortic and pulmonary flow measurements were previously often reported as repeatable measurements $[25,28,29]$, except in one study on horses in which they were poorly repeatable [27]. The lack of repeatability of the pulmonary flow in the studied goats could be explained by the difficulty to obtain a good quality $2 \mathrm{D}$ image of the right ventricular outflow tract, then to place the sample volume day to day exactly at the good location. Recordings of the pulmonary flow from a left parasternal cranial long axis right ventricular outflow view or from a left parasternal short axis view with aorta and pulmonary artery, as used in small domestic animals $[10,25,29]$, has not been investigated in this study but appeared to be difficult to obtain in goats.

The variability of the PW Doppler measurements in our study was quite high, but was in accordance with previous studies in dogs, cats and horses $[7,25,26,29]$. Independently of the valve where the blood flow was measured, the within-day and between-day $\mathrm{CV}$ ranged from $4.68 \%$ to $41.53 \%$, but most of these were inferior to $20 \%$ (Tables 1, 2, 3, 4). The main source of variability could be the poor alignment of the transducer with the direction of the blood flow [10,26,27]. Measurements of the Acc or Dec slopes and times from the different blood flows were the most variable parameters. This is in agreement with previous studies in horses [26] and in dogs [25].

In the studied goats, the mean values of the mitral velocity spectrum obtained from a tilted left parasternal long axis four chamber view were significantly different from those obtained from a tilted right parasternal long axis four chamber view, except for all parameters of the A peak and for $\mathrm{ET}$ of the $\mathrm{E}$ peak. Moreover, $\mathrm{E}_{\max }$ and $\mathrm{E}_{\text {mean }}$ were lower when the measurements were performed from the right side than from the left side, which suggests that in goats, the mitral flow should be interrogated from the left rather than from the right hemi thorax. This result is in agreement with previous studies on other domestic animals since, to record the mitral flow, a tilted left parasternal long axis four chambers view is recommended in horses [7], and a left parasternal apical view is recommended in sheep [9] and in dogs [4].

The $E_{\max } / A_{\max }$ ratio is a parameter often used to evaluate the left ventricular diastolic function in man $[2,30]$. Independently of the side from which it is measured, the $E_{\max } / A_{\max }$ ratio of the mitral flow was rather similar to the tricuspid flow $E_{\max } / A_{\max }$. On the contrary to what was reported in sheep [9], in most goats of this study, $E_{\max }$ was higher than $A_{\max }$ for both mitral and tricuspid flows, and only one goat had $E_{\max } / A_{\max }<1$ for mitral flow obtained from the right side. The same was observed in 8 of 40 investigated healthy horses [7] and was explained as a more accurate alignment of the 
transducer with the A wave of atrial contraction than with the $E$ wave of the early rapid ventricular filling. Measurements of E peak and A peak seemed also to depend on HR. In goats as in sheep, it has been reported than the A peak is closer to the $\mathrm{E}$ peak with increasing $\mathrm{HR}$, and when HR was more than 120 beats/min, fusion of the two peak can occur [9].

Measurements of aortic velocity spectrum are very interesting because they allow assessing left ventricular SV and CO [2]. In this study, except for PEP and PEP/ ET, the aortic velocity spectrum measurements obtained from the tilted left parasternal long axis five chambers view were significantly higher than those obtained from a tilted right parasternal long axis five chambers view. This is in agreement with the results obtained in horses [7], and could be explained by a better alignment between the transducer and the blood flow from the left hemi thorax. This could also explain why previous measurements of aortic flow parameters reported in Swedish goats and obtained from the right side [18] were lower than those obtained in this present study.

\section{Conclusions}

PW-Doppler flow parameters reference values in adult, healthy, non-pregnant, female goats of the Saanen breed are reported in this study. As documented in other species, goats' PW Doppler parameters measurements showed a poor between-day repeatability and a high but acceptable within-day and between-day variability. Tricuspid and pulmonary flows should be evaluated from the right hemi thorax whereas mitral and aortic flows should be obtained from the left hemi thorax. Moreover, in the future, it could be interesting to evaluate the effect of physiological factors in order to establish fully documented PW Doppler echocardiographic reference values in goats.

\section{Competing interests}

The authors declare that they have no competing interests.

\section{Authors' contributions}

Conception and design of the study: AAL, FF and HA; Data acquisition: AAL; statistical analysis: AAL, FF; Drafting and critically revising the manuscript: AAL, FF, MLM, CFS, SD and HA. All authors read and approved the final manuscript.

\section{Acknowledgements \\ This work has been performed at the Equine Clinic, Faculty of veterinary Medicine, University of Liege, in collaboration with the Department of Cardiology, University Hospital of Liege, Liege - Sart Tilman, Belgium, and has been supported by the French Community of Belgium (Actions de Recherches Concertées - Académie Wallonie-Europe) \\ Acknowledgments to Vincent Verleyen and Sylvain Romijn for their technical assistance.}

\section{Author details}

${ }^{1}$ Equine Clinic, Department of Companion Animals and Equids, Faculty of Veterinary Medicine, University of Liege, Boulevard de Colonster 20, Bât B41, Liege, Sart Tilman, Belgium. ${ }^{2}$ Biostatistics, Bioinformatics and Animal Selection, Department of Animal Production, Faculty of Veterinary Medicine,
University of Liege, Boulevard de Colonster 20, Bât B43, Liege, Sart Tilman, Belgium. ${ }^{3}$ Department of Cardiology, University Hospital of Liege, Medicine Faculty, University of Liege, Avenue de I'Hôpital 13, Bât B35, Liege, Sart Tilman, Belgium.

Received: 19 March 2012 Accepted: 12 October 2012 Published: 16 October 2012

\section{References}

1. Gardin JM, Burn CS, Childs WJ, Henry WL: Evaluation of blood flow velocity in the ascending aorta and main pulmonary artery of normal subjects by Doppler echocardiography. Am Heart J 1984, 107:310-319.

2. Anderson B: Echocardiography: The Normal Examination and Echocardiographic Measurements. 2nd edition. Brisbane: MGA Graphics; 2007.

3. Abbott JA, MacLean HN: Comparison of Doppler-derived peak aortic velocities obtained from subcostal and apical transducer sites in healthy dogs. Vet Radiol Ultrasound 2003, 44:695-698.

4. Yuill CD, O'Grady MR: Doppler-derived velocity of blood flow across the cardiac valves in the normal dog. Can J Vet Res 1991, 55:185-192.

5. Santilli RA, Bussadori C: Doppler echocardiographic study of left ventricular diastole in non-anaesthetized healthy cats. Vet J 1998, 156:203-215.

6. Reef VB, Lalezari K, De Boo J, van der Belt AJ, Spencer PA, Dik KJ: Pulsedwave Doppler evaluation of intracardiac blood flow in 30 clinically normal Standardbred horses. Am J Vet Res 1989, 50:75-83.

7. Blissitt KJ, Bonagura JD: Pulsed wave Doppler echocardiography in normal horses. Equine Vet J 1995, 27(Suppl 19):38-46.

8. Hallowell GD, Potter TJ, Bowen IM: Methods and normal values for echocardiography in adult dairy cattle. J Vet Cardiol 2007, 9:91-98.

9. Kirberger RM, Berg JS: Pulsed wave Doppler echocardiographic evaluation of intracardiac blood flow in normal sheep. Res Vet Sci 1993, 55:189-194.

10. Boon J: Veterinary Echocardiography. 2nd edition. Chichester: Wiley-Blackwell; 2011.

11. Marr CM, Patteson MW: Echocardiography. In Cardiology of the Horse. 2nd edition. Edited by Marr CM, Bowen M. Edinburgh, London, New York, Oxford, Philadelphia, St-Louis, Sydney, Toronto: Saunders Elsevier; 2010:105-126.

12. Remes J, van Brakel TJ, Bolotin G, Garber C, de Jong MM, van der Veen FH, Maessen JG: Persistent atrial fibrillation in a goat model of chronic left atrial overload. J Thorac Cardiovasc Surg 2008, 136:1005-1011.

13. Brice AG, Forster HV, Pan LG, Brown DR, Forster AL, Lowry TF: Effect of cardiac denervation on cardiorespiratory responses to exercise in goats. J Appl Physiol 1991, 70:1113-1120.

14. Acorda JA, Ong RAF, Maligaya RL: Ultrasonographic features of the heart in Philippine native goats (Capra hircus). Philipp J Vet Med 2005, 42:66-74.

15. Gardner SY, Reef VB, Palmer JE, Reimer JM, Sweeney RW: Echocardiographic diagnosis of an anomaly of the tricuspid valve in a male pygmy goat. J Am Vet Med Assoc 1992, 200:521-523.

16. Leroux AA, Moonen ML, Farnir F, Sandersen CF, Deleuze S, Salciccia A, Amory $\mathrm{H}$ : Two-dimensional and $\mathrm{M}$-mode echocardiographic reference values in healthy adult Saanen goats. Vet Rec 2012, 170:154.

17. Steininger K, Berli AS, Jud R, Schwarzwald CC: Echocardiography in Saanen-goats: Normal findings, reference intervals in awake goats, and the effect of general anesthesia. Schweiz Arch Tierheilkd 2011, 153:553-564.

18. Olsson K, Hansson A, Hydbring E, von Walter LW, Haggstrom J: A serial study of heart function during pregnancy, lactation and the dry period in dairy goats using echocardiography. Exp Physiol 2001, 86:93-99.

19. Thomas WP, Gaber CE, Jacobs GJ, Kaplan PM, Lombard CW, Moise NS, Moses BL: Recommendations for standards in transthoracic twodimensional echocardiography in the dog and cat. Echocardiography Committee of the Specialty of Cardiology, American College of Veterinary Internal Medicine. J Vet Intern Med 1993, 7:247-252.

20. Kirberger RM, Bland-Van Den Berg P, Grimbeek RJ: Doppler echocardiography in the normal dog: Part II. Factors influencing blood flow velocities and a comparison betwen left and right heart blood flow. Vet Radiol Ultrasound 1992, 33:380-386.

21. Al Haidar A, Farnir F, Deleuze S, Sandersen C, Amory H: Comparison of the Repeatability of Echocardiographic Measurements from Different Modes and Views in Horses of Various Breeds and Sizes. J Equine Vet Sci 2010, 30:287-297. 
22. Disatian S, Bright JM, Boon J: Association of age and heart rate with pulsed-wave Doppler measurements in healthy, nonsedated cats. J Vet Intern Med 2008, 22:351-356.

23. Mesa A, Jessurun C, Hernandez A, Adam K, Brown D, Vaughn WK, Wilansky $S$ : Left ventricular diastolic function in normal human pregnancy. Circulation 1999, 99:511-517.

24. Long K, Bonagura JD, Darke PG: Standardised imaging technique for guided M-mode and Doppler echocardiography in the horse. Equine Vet J 1992, 24:226-235.

25. Dukes-McEwan J, French AT, Corcoran BM: Doppler echocardiography in the dog: Measurement variability and reproducibility. Vet Radiol Ultrasound 2002, 43:144-152.

26. Young LE, Scott GR: Measurement of cardiac function by transthoracic echocardiography: day to day variability and repeatability in normal Thoroughbred horses. Equine Vet J 1998, 30:117-122.

27. Buhl R, Ersboll AK, Eriksen L, Koch J: Sources and magnitude of variation of echocardiographic measurements in normal standardbred horses. Vet Radiol Ultrasound 2004, 45:505-512.

28. Kriz NG, Rose RJ: Repeatability of standard transthoracic echocardiographic measurements in horses. Aust Vet J 2002, 80:362-370.

29. Simpson KE, Devine BC, Gunn-Moore DA, French AT, Dukes-McEwan J, Koffas H, Moran CM, Corcoran BM: Assessment of the repeatability of feline echocardiography using conventional echocardiography and spectral pulse-wave Doppler tissue imaging techniques. Vet Radiol Ultrasound 2007, 48:58-68.

30. Demaria AN, Wisenbaugh TW, Smith MD, Harrison MR, Berk MR: Doppler Echocardiographic Evaluation of Diastolic Dysfunction. Circulation 1991, 84:1288-1295.

doi:10.1186/1746-6148-8-190

Cite this article as: Leroux et al:: Repeatability, variability and reference values of pulsed wave Doppler echocardiographic measurements in healthy Saanen goats. BMC Veterinary Research 2012 8:190.

\section{Submit your next manuscript to BioMed Central and take full advantage of:}

- Convenient online submission

- Thorough peer review

- No space constraints or color figure charges

- Immediate publication on acceptance

- Inclusion in PubMed, CAS, Scopus and Google Scholar

- Research which is freely available for redistribution 